\title{
Delineation of the genus Actinobacillus by comparison of partial infB sequences
}

\author{
Niels Nørskov-Lauritsen, ${ }^{1,2}$ Henrik Christensen, ${ }^{3}$ Henrik Okkels, ${ }^{4}$ \\ Mogens Kilian ${ }^{2}$ and Brita Bruun ${ }^{5} \dagger$ \\ 1,4 Departments of Clinical Microbiology ${ }^{1}$ and Clinical Biochemistry ${ }^{4}$, Aalborg Hospital, Aalborg,
Denmark
${ }^{2}$ Department of Medical Microbiology and Immunology, University of Aarhus, Aarhus, Denmark
${ }^{3}$ Department of Veterinary Microbiology, The Royal Veterinary and Agricultural University, Copenhagen, Denmark
${ }^{5}$ Department of Clinical Microbiology, State Serum Institute, Copenhagen, Denmark

Niels Nørskov-Lauritsen

norskov@microbiology.au.dk

\begin{abstract}
A 426 bp fragment of infB, a housekeeping gene that encodes translation initiation factor 2, was sequenced from 59 clinical isolates and type strains of Actinobacillus species and sequences were compared. Partial sequences of $16 \mathrm{~S}$ rRNA genes were also obtained. By comparing infB sequences, Actinobacillus pleuropneumoniae, Actinobacillus equuli, Actinobacillus suis, Actinobacillus ureae, Actinobacillus arthritidis, Actinobacillus hominis and two unnamed genomospecies showed more than $85 \%$ similarity to the type strain of the type species of the genus, Actinobacillus lignieresii. The taxonomic position of Actinobacillus capsulatus was unresolved; this species is more remotely related to $A$. lignieresii. The two species $A$. lignieresii and $A$. pleuropneumoniae could not be clearly separated by infB sequence analysis. The phylogeny of the genus Actinobacillus based on infB analysis was essentially congruent with relationships inferred from 16S rRNA sequence comparisons and DNA hybridization studies. Discrepancies were encountered with single strains or taxa at the periphery of the genus. Greater intraspecies variation was observed with infB sequences than with $16 \mathrm{~S}$ rRNA gene sequences, with notable exceptions. The apparent subdivision of some species by $16 \mathrm{~S}$ rRNA analysis was most likely caused by RNA operon heterogeneity.
\end{abstract}

\section{INTRODUCTION}

The generic name Actinobacillus was introduced by Brumpt in 1910 with the binomen Actinobacillus lignieresii. He referred to 'l'actinobacille' associated with characteristic chronic granulomatous lesions in cattle that was described by Lignieres and Spitz in 1902. Five species were listed as members of the genus in the 1984 edition of Bergey's Manual of Systematic Bacteriology: A. lignieresii, Actinobacillus equuli, Actinobacillus suis, Actinobacillus capsulatus and Actinobacillus actinomycetemcomitans (Phillips, 1984). In the most recent edition, the number of species was 18 , of which only a few are considered to belong to Actinobacillus sensu stricto (Olsen \& Møller, 2004).

tPresent address: Department of Clinical Microbiology, Hillerød Hospital, Hillerød, Denmark.

The GenBank/EMBL/DDBJ accession numbers for the sequences reported in this paper are AJ438097-AJ438128, AJ438436AJ438462 and AJ438630-AJ438688.

A matrix table of infB sequence similarity values is available as supplementary material in IJSEM Online.
DNA hybridization results from the genus Actinobacillus have been presented by several groups. Based on the spectrophotometric method, Mutters et al. (1989) suggested that the genus Actinobacillus should be restricted to A. lignieresii, Actinobacillus pleuropneumoniae, A. equuli, A. suis, A. capsulatus, Actinobacillus ureae, Actinobacillus hominis and Bisgaard taxa 5, 9 and 11. This delineation was presented in the form of a review, and crucial information, including the number of strains and the individual DNA binding values, was not given. In a study using the S1 nuclease method on multiple strains from several species, A. equuli, A. suis, A. ureae and A. capsulatus were sufficiently related to $A$. lignieresii to justify their inclusion in the genus (Escande et al., 1984). A single isolate received as A. equuli was unexpectedly found to differ from the other representatives of this species, but was located within the boundaries of the genus by DNA hybridization. This strain was later found to comply with Bisgaard taxon 9 and has been made the type strain of a novel species, Actinobacillus arthritidis (Christensen et al., 2002a). Bisgaard taxon 11 is phenotypically a haemolytic $A$. equuli, closely related to the type strain of $A$. equuli by DNA hybridization, and the 
taxon has been reclassified as A. equuli subsp. haemolyticus (Christensen et al., 2002b).

Increasing emphasis has been directed to the division of bacteria into groups based on comparison of 16S rRNA sequences - so-called phylogenetic classification (Krieg \& Garrity, 2001). Based on a large number of near-fulllength 16S rRNA sequences from different members of the family Pasteurellaceae, single representatives of the following species or taxa constitute a monophyletic group: A. lignieresii, A. pleuropneumoniae, A. equuli, A. suis, A. ureae, A. hominis, A. arthritidis, Bisgaard taxon 8 and strain CCUG 28015 (a duck isolate). The other Actinobacillus species are pending placement in other genera of the family Pasteurellaceae (Olsen et al., 2004).

Translation initiation factor 2 is an essential protein involved in the initiation of protein synthesis in prokaryotes. So far, the encoding gene, inf $B$, has only been found as a single copy in the prokaryotic genome; it encompasses $2490 \mathrm{bp}$ in Haemophilus influenzae (Fleischmann et al., 1995) and 2502 bp in Pasteurella multocida (May et al., 2001). A partial sequence of $\inf B$ has been used successfully in a study of the genus Haemophilus (Hedegaard et al., 2001). In the present study, the investigation was extended to the genus Actinobacillus. The principal scope was species that have qualified as true members of the genus by DNA hybridization or $16 \mathrm{~S}$ rRNA sequencing. The following were included: 11 representatives of the type species of the genus, $A$. lignieresii, four of which were equine isolates that appear to constitute a new genomospecies (genomospecies 1) of Actinobacillus that is distinct from A. lignieresii (Christensen et al., 2002a); representatives of species belonging to the genus by both DNA hybridization and rRNA sequence data (A. pleuropneumoniae, A. equuli, A. suis, A. ureae, A. hominis and A. arthritidis); representatives of the sorbitolnegative subgroup of Bisgaard taxon 9 (genomospecies 2), which belong to the genus according to molecular data (Christensen et al., 2002a); representatives of A. capsulatus and Bisgaard taxon 5, which are included in the genus by DNA hybridization data, but excluded on the basis of rRNA sequencing; and a few single isolates that are candidate species of the genus by $16 \mathrm{~S}$ rRNA sequence comparison (CCUG 28015 and Bisgaard taxon 8) or can be used as outgroups (Actinobacillus muris and two biotypes of Pasteurella pneumotropica).

\section{METHODS}

Bacterial strains and DNA preparation. The bacterial strains sequenced in this study are listed in Table 1. DNA was released using the chelex method as described previously (Hedegaard et al., 2001). PCR products were purified on silica gel columns (QIAquick PCR purification kit; Qiagen).

infB sequencing. Fragments of inf $B$ spanning approximately $650 \mathrm{nt}$ were amplified using the degenerate oligonucleotide primers $1186 \mathrm{f}$ and 1833r (Hedegaard et al., 1999). A total of 30 cycles was run, each consisting of a denaturation step at $94{ }^{\circ} \mathrm{C}$ for $30 \mathrm{~s}$, an annealing step at $51^{\circ} \mathrm{C}$ for $60 \mathrm{~s}$ and an elongation step at $72{ }^{\circ} \mathrm{C}$ for
90 s. Purified products were cycle-sequenced using the non-degenerate internal primer 1748R (5'-GTAGCAACCGGACCACGACCTTTAT$\left.3^{\prime}\right)$ and the BigDye terminator DNA sequencing kit (Applied Biosystems); analysis was on an ABI 377 DNA sequencer. Primers are named according to nucleotide positions in the infB gene of Escherichia coli (Hedegaard et al., 1999). The corresponding positions in the P. multocida gene are nt 1018, 1580 and 1665 (May et al., 2001). With A. arthritidis strain CCUG $24862^{\mathrm{T}}$ and P. pneumotropica strain $\mathrm{P} 309$, a probable $3^{\prime}$ mismatch prevented use of the internal primer, and sequencing was performed with the primers used for PCR amplification. A $426 \mathrm{bp}$ fragment was obtained corresponding to amino acid positions 368-509 [with reference to $P$. multocida translation initiation factor 2 (May et al., 2001)]; sequences of all bacterial strains were compared. Sequences were checked by visual examination of the electrophoretograms, by translation into amino acid sequences and finally by re-examination of polymorphic positions within individual species or taxa. Ambiguities were resolved by repeating the PCR and sequencing reactions and by sequencing in both directions with the primers used for PCR amplification.

$16 S$ rRNA gene sequencing. Fragments of the 16S rRNA genes were amplified by PCR using primers $5^{\prime}$-TATTACCGCGGCTGCTGGCA- $3^{\prime}$ and $5^{\prime}$-TCAGATTGAACGCTGGCGGC- $3^{\prime}$ derived from the $r r n B$ operon of E. coli (Brosius et al., 1978). A total of 25 cycles was run, each consisting of a denaturation step at $94{ }^{\circ} \mathrm{C}$ for $60 \mathrm{~s}$, an annealing step at $60^{\circ} \mathrm{C}$ for $60 \mathrm{~s}$ and an elongation step at $72{ }^{\circ} \mathrm{C}$ for 2 min. Purified products were cycle-sequenced from both ends using the PCR primers and a ThermoSequenase dye terminator sequencing kit (Pharmacia Biotech); analysis was on an ABI 377 DNA sequencer. Fragments of $429-437$ bp (corresponding to nt 62-496 of E. coli 16S rRNA; GenBank/EMBL accession no. J01695) were obtained from all bacterial strains and used for analysis.

An unresolved nucleotide in one sequencing direction was ignored if the complementary position was determined unambiguously. If the position was determined ambiguously in both sequencing directions, the sequencing reactions were repeated. If the ambiguity persisted after the second round of reactions, no further resolution attempt was carried out.

Sequence analysis. Sequences were aligned using the program CLUSTAL W with a Windows interface (Thompson et al., 1997). Distances were modified by the Kimura two-parameter method (Kimura, 1980). Neighbour-joining, maximum-parsimony and maximum-likelihood analysis were performed by PHYLIP (Felsenstein, 1995). Bootstrap analyses were done by 100 resamplings of the datasets. The calculation of similarity between clusters (see matrix table available as supplementary material in IJSEM Online) or sequences (Table 2) was performed with even-weighted characters. When $16 \mathrm{~S}$ rRNA was compared, gaps and ambiguous positions were ignored.

\section{RESULTS}

\section{Comparison of infB sequences}

A 426 bp fragment of infB was unambiguously determined from the 59 bacterial strains seen in Table $1 ; 163$ polymorphic positions were available for phylogenetic analysis. Compared with the type strain of the type species of the genus (A. lignieresii $\mathrm{P} 151^{\mathrm{T}}$ ), 2613 nucleotide substitutions were present in the 58 other strains; when the protein sequences were compared, a total of 214 amino acid substitutions were detected.

A dendrogram constructed by the neighbour-joining 
Table 1. Strains of Actinobacillus and Pasteurella included in this study

Culture designations and abbreviations: ATCC, American Type Culture Collection, Manassas, VA, USA; CCUG, Culture Collection, University of Göteborg, Göteborg, Sweden; F, from the collection of Magne Bisgaard, The Royal Veterinary and Agricultural University, Copenhagen, Denmark; HK, from the collection of M. K.; NCTC, National Collection of Type Cultures, Colindale, London, UK; P, from the collection of Wilhelm Frederiksen, Department of Clinical Microbiology, State Serum Institute, Copenhagen, Denmark.

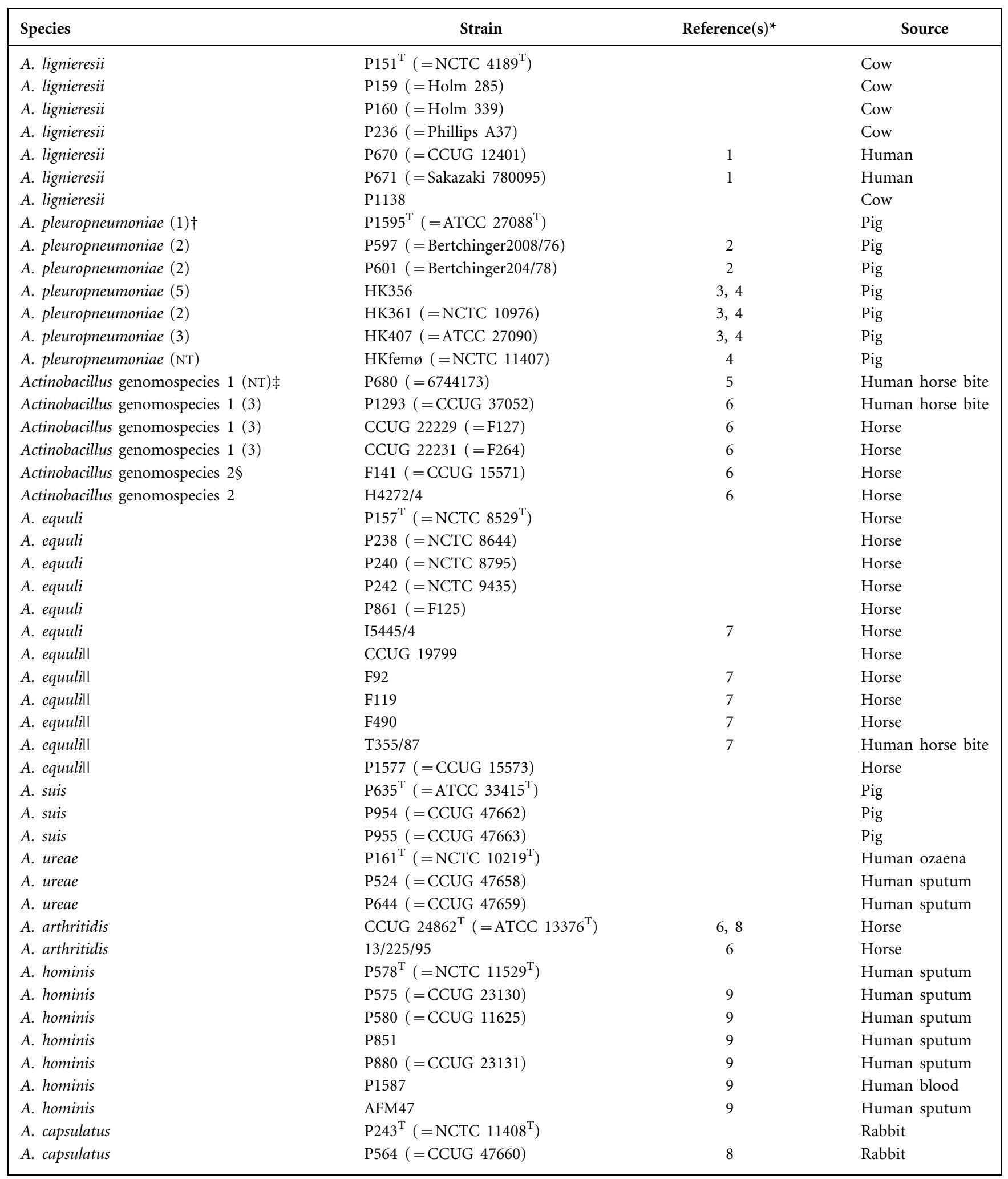


Table 1. cont.

\begin{tabular}{|c|c|c|c|}
\hline Species & Strain & Reference $(s)^{*}$ & Source \\
\hline A. capsulatus & P796 (=CCUG 47661) & 10 & Rabbit \\
\hline Bisgaard taxon 8 & P1572 (= CCUG 16494) & & Guinea pig \\
\hline P. pneumotropica Jawetz & $\mathrm{P} 421^{\mathrm{T}}\left(=\right.$ NCTC $\left.8141^{\mathrm{T}}\right)$ & & Mouse \\
\hline Bisgaard taxon 5 & CCUG 16493 & & Guinea pig \\
\hline Bisgaard taxon 5 & CCUG 24854 & & Guinea pig \\
\hline A. muris & $\mathrm{P} 1573^{\mathrm{T}}\left(=\right.$ CCUG $\left.16938^{\mathrm{T}}\right)$ & & Mouse \\
\hline
\end{tabular}

${ }^{\star}$ 1, Fussing et al. (1998); 2, Pohl et al. (1983); 3, Kilian (1976); 4, Kilian et al. (1978); 5, Dibb et al. (1981); 6, Christensen et al. (2002a); 7, Christensen et al. (2002b); 8, Escande et al. (1984); 9, Friis-Møller et al. (2001); 10, Olsen et al. (2004).

$\dagger$ Numbers in parentheses refer to serotypes of H. pleuropneumoniae. Typing was also performed with strains of Actinobacillus genomospecies 1. NT, Not typable.

¥Equine isolates phenotypically identified as A. lignieresii.

§Sorbitol-negative subgroup of Bisgaard taxon 9.

IIHaemolytic variant; A. equuli subsp. haemolyticus according to Christensen et al. (2002b).

method is shown in Fig. 1, with 52 strains clustering in nine sequence groups. Phylogenetic trees were also generated by the maximum-parsimony and maximum-likelihood methods (dendrograms not shown). These analyses generally supported the major topology of Fig. 1; some contradictory findings are mentioned in the text.

\section{A. lignieresii branch}

Twenty strains, encompassing clusters 1,2 and 3 from Fig. 1, were placed on a common branch by all three phylogenetic methods. The branch was supported by a moderate bootstrap value of 69 (of 100 resamplings) (Fig. 1) and relatively large differences were observed between individual clusters in the branch. The branch contained A. lignieresii, A. pleuropneumoniae and two unnamed taxa.

Fourteen strains of A. lignieresii and A. pleuropneumoniae, including the two type strains, were found in a common cluster with a sequence similarity in excess of $96 \%$ (cluster 1). The cluster was further divided into four subgroups, with some distinction between A. lignieresii and A. pleuropneumoniae: one subgroup included only strains of $A$. lignieresii and two subgroups contained only A. pleuropneumoniae. In a fourth subgroup, a strain of A. pleuropneumoniae (HK356) carried an infB sequence that was almost identical to that of the type strain of A. lignieresii (Fig. 1).

Four equine isolates phenotypically identified as A. lignieresii constituted cluster 2 . These were clearly distinct from the type strain and the other bovine isolates of A. lignieresii located in cluster 1 . However, these isolates were related to strains in cluster 1 (similarity of 89-92\%). Equine isolates of $A$. lignieresii have recently been described as a unique genomospecies (genomospecies 1) based on DNA hybridization and 16S rRNA gene sequencing (Christensen et al., 2002a). Two other equine isolates, F141 and H4272/4, constituted a separate cluster on the A. lignieresii branch (cluster 3 ). These isolates comply phenotypically with the sorbitol-negative subgroup of Bisgaard taxon 9 (genomospecies 2) (Christensen et al., 2002a).

\section{A. equuli branch}

Six clusters (4-9) representing A. equuli, A. suis, A. ureae, A. arthritidis, A. hominis and A. capsulatus were placed on a common branch by the neighbour-joining method (Fig. 1). The branch was supported by a bootstrap value of 64 . The A. capsulatus cluster (cluster 9) branched rather deeply and its location on the A. equuli branch was not confirmed by maximum-parsimony or maximum-likelihood analyses (dendrograms not shown).

Twelve bacterial strains representing $A$. equuli and its haemolytic variants constituted cluster 4 . The latter phenotype has been designated Bisgaard taxon 11 or A. equuli subsp. haemolyticus (Christensen et al., 2002b). Although some division of the cluster into subgroups was apparent, subgrouping according to inf $B$ genotype did not correlate with haemolytic activity. A bootstrap value of only 20 was found for cluster 4 , indicating difficulties in the distinction between $A$. equuli and $A$. suis (cluster 5 ). The three representatives of $A$. suis carried almost identical infB sequences, with only a single polymorphic position found in the studied fragment. A similar situation was found in the three representatives of $A$. ureae (cluster 6); however, these strains were more clearly separated from sequences in the A. equuli cluster. A similarity of $95-97 \%$ was found between clusters 4 and 6 , whereas the differences between clusters 4 and 5 were of the same magnitude as the differences found within cluster 4 (see supplementary material in IJSEM Online). 


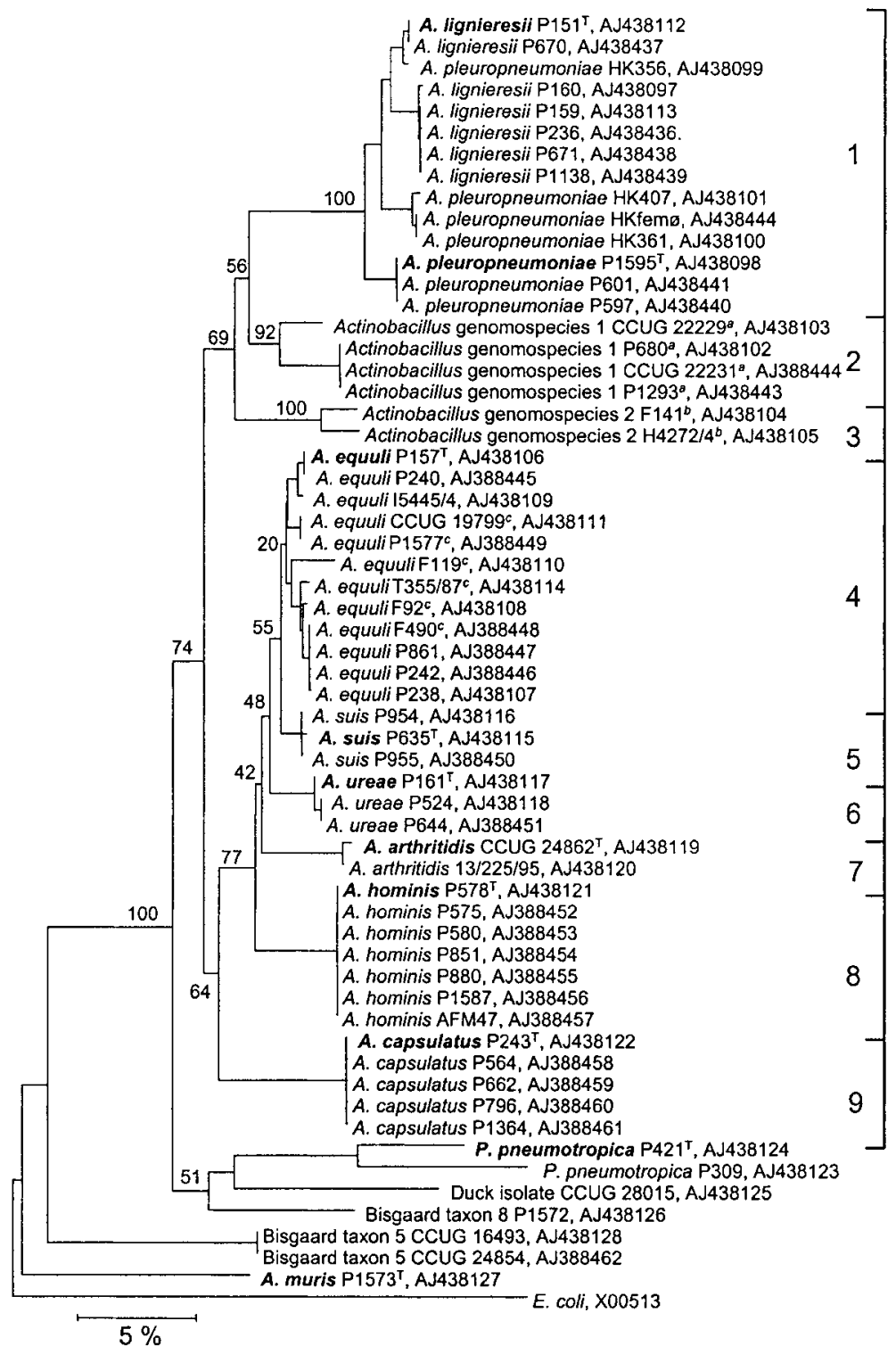

Fig. 1. Neighbour-joining nucleotide distance tree based on partial sequences of infB with evolutionary distances given in terms of base substitutions weighted by the Kimura twoparameter model. Type strains in bold and nucleotide sequence accession numbers are given. Values to the left of nodes are percentages of bootstrap replications supporting the node. The infB sequence from $E$. coli was used as the outgroup. Superscript letters are defined as follows: $a$, equine isolates phenotypically identified as $A$. lignieresii; $b$, sorbitol-negative subgroup of Bisgaard taxon $9 ; c$, haemolytic variant $(A$. equuli subsp. haemolyticus according to Christensen et al., 2002b).

Two representatives of the newly described species $A$. arthritidis were positioned on the A. equuli branch by all three phylogenetic methods (cluster 7). This species was formed from the sorbitol-positive subgroup of Bisgaard taxon 9 (Christensen et al., 2002a).

Identical $\inf B$ sequences were observed in seven strains of A. hominis (cluster 8) and in five strains of A. capsulatus (cluster 9). The relationship of A. hominis to clusters 4-7 was supported by a bootstrap value of 77 and the topology was also seen in dendrograms constructed by maximumparsimony and maximum-likelihood analyses (not shown). The location of the A. capsulatus cluster was more questionable, as the two other phylogenetic methods placed this cluster on another branch together with the group consisting of P. pneumotropica $\left(\mathrm{P} 421^{\mathrm{T}}\right), \mathrm{P} 309$, CCUG 28015 and P1572; indeed, by maximum-likelihood analyses, the A. capsulatus cluster was even less related to the 'true' actinobacilli (clusters 1-8) than was the P. pneumotropica group. The simple index of similarity also reveals the uncertain position of the A. capsulatus cluster, as this cluster (cluster 9) differed more from the A. lignieresii cluster (cluster 1) than from the other groups of actinobacilli (see supplementary material in IJSEM Online).

\section{Strains more remotely related to the genus Actinobacillus}

Compared to the other strains studied, the type strain of $P$. pneumotropica and an additional strain (biotype Heyl, P309), a duck isolate (CCUG 28015), one representative of Bisgaard taxon 8 (P1572), two representatives of Bisgaard taxon 5 (CCUG 16493 and CCUG 24854) and the type strain of $A$. muris were more remotely related to the type species of the genus Actinobacillus using infB sequence analysis (Fig. 1). 


\section{Comparison of partial 16S rRNA gene sequences}

A fragment from the $5^{\prime}$ end of the $16 \mathrm{~S}$ rRNA gene was sequenced from all 59 bacterial strains included in the present study. The fragment encompassed $437 \mathrm{nt}$ in strains of A. lignieresii and A. pleuropneumoniae (cluster 1); $435 \mathrm{nt}$ in clusters 2-9, CCUG 28015, P1572 and the two representatives of Bisgaard taxon 5; $431 \mathrm{nt}$ in P. pneumotropica biotype Heyl; and $429 \mathrm{nt}$ in the type strains of $P$. pneumotropica and A. muris (GenBank/EMBL accession nos AJ438630-AJ438688). Phylogenetic analysis based on near-full-length $16 \mathrm{~S}$ rRNA sequences from 114 representatives of the family Pasteurellaceae has recently been published (Olsen et al., 2004), so the 16S rRNA sequencing in the present study was carried out to ascertain the identity of the isolates and to study intraspecies variation. In most cases, minor differences were found between the sequence of the rRNA gene fragment determined by us and the sequences available in the databases for the same strains. The majority of discrepancies concerned single bases that were either resolved in our sequence, but undetermined in GenBank/EMBL, or vice versa.

The subgrouping of cluster 1 observed by infB analysis was not supported by $16 \mathrm{~S}$ rRNA gene sequencing. Nearfull-length $16 \mathrm{~S}$ rRNA sequences obtained with the type strains of A. lignieresii and A. pleuropneumoniae were almost identical and differed in only 4 nt positions (Fussing et al., 1998). Two of the polymorphic positions occurred in the fragment used in the present study and these two signature bases were repeatedly confirmed in the rRNA sequence of the other 12 strains of infB cluster 1, allowing a distinction of A. lignieresii from A. pleuropneumoniae. The variation within the two species was restricted to a single polymorphic position in each species $(0.2 \%)$ in the fragment of the 16S rRNA gene studied (GenBank/EMBL accession nos AJ438630-AJ438642 and AJ438688; alignment not shown).

With the five representatives of $A$. capsulatus, three distinct sequences were obtained (GenBank/EMBL accession nos AJ438675-AJ438679). The previously published sequences of the type strain and P796 were essentially confirmed, whereas the three remaining isolates carried mutually identical 16S rRNA gene fragment sequences with $98.6 \%$ similarity to the type strain of the species.

Relatively large differences between the multiple $r r n$ operons were indicated in some species of the genus Actinobacillus. With the type strain of $A$. hominis and an additional strain (P575, GenBank/EMBL accession nos AJ438666-AJ438667), the nucleotides GCAA were found in positions where the $\mathrm{rrnB}$ operon of E. coli carries the sequence TTCG (nt 208211 , numbering with reference to $E$. coli $16 \mathrm{~S}$ rRNA). With the five other isolates of $A$. hominis, the sequencing in this area was ambiguous, as two distinct nucleotides at each position were discernible in both sequencing directions. The 16S rRNA sequence of the type strain, available as L06076, recognizes the ambiguity at position 208, but not at
209-211. With all seven representatives of A. hominis (GenBank/EMBL accession nos AJ438666-AJ438672), as well as the deposited sequence (L06076), positions 199 and 218 were ambiguously determined.

Representatives of $A$. equuli were divided into two groups, one group being almost identical to $A$. suis and the other showing more resemblance to $A$. hominis. These separate sequence groups have been previously represented as GenBank/EMBL accession numbers M75072 (the type strain of $A$. equuli) and M75067 (CCUG 19799). The latter strain has erroneously been referred to as A. capsulatus (Dewhirst et al., 1992), but it is a haemolytic variant of A. equuli, formerly designated Bisgaard taxon 11 (Berthoud et al., 2002; Christensen et al., 2002b). Haemolytic activity was not correlated with $16 \mathrm{~S}$ rRNA sequence, as both groups contained haemolytic as well as non-haemolytic strains (dendrogram not shown). In the fragment of 16S rRNA studied, the difference between the two groups of $A$. equuli was determined by 12 polymorphic positions (nt 456-461 and 471-476, E. coli numbering). However, with the type strain of $A$. equuli $\left(\mathrm{P} 157^{\mathrm{T}}\right.$, GenBank/EMBL accession no. AJ438649), electrophoretograms were obtained from both sequencing directions that were clearly an average of the two sequences at the 12 polymorphic positions. The sequencing was repeated with bacteria subcultured twice from a single colony to ascertain the purity of the strain and the same results were obtained.

\section{Delineation of the genus Actinobacillus by different molecular methods}

In Table 2, the results of partial infB sequencing are shown along with results obtained by $16 \mathrm{~S}$ rRNA gene sequencing and DNA hybridization data. The type strains of different species (or reference strains from unnamed taxa) are compared with the type strain of the type species of the genus, A. lignieresii. The results from DNA hybridization experiments were compiled from two separate sources and are incomplete. Gaps and ambiguous positions are ignored in the calculation of rRNA similarity.

The results from DNA hybridization are not precise. Rather different results were obtained using the two methods with the type strain of $A$. suis or using the same method on highly related biovars of $A$. equuli (Table 2). The DNA relationship is high between the type strain of $A$. lignieresii and the reference strain of equine lignieresii (genomospecies 1, CCUG 22229), but decreases to a level of around $40 \%$ in relation to members of the A. equuli branch. With $16 \mathrm{~S}$ rRNA gene comparison, 97·0-98.3\% similarity was seen between the type strains of $A$. equuli, A. suis, A. ureae, $A$. arthritidis and $A$. hominis (all of the $A$. equuli branch) and A. lignieresii. A similar level was observed with Bisgaard taxon 8, whereas the duck isolate CCUG 28015 was as different from $A$. lignieresii as Bisgaard taxon $5(95 \cdot 1-$ $95.7 \%$ ), which is unclustered by phylogenetic analysis (Olsen et al., 2004). A conspicuous result is seen with the type strain of $A$. capsulatus, which is about as different from 
Table 2. Delineation of the genus Actinobacillus as determined by DNA hybridization plus $16 \mathrm{~S}$ rRA and partial infB sequences

Columns headed Escande and Christensen respectively give data from Escande et al. (1984) and Christensen et al. (2002a). -, Not reported.

\begin{tabular}{|c|c|c|c|c|c|}
\hline Strain & $\begin{array}{l}\text { 16S rRNA gene } \\
\text { accession no. }\end{array}$ & \multicolumn{4}{|c|}{ Similarity to the type strain of $A$. lignieresii (\%) } \\
\hline A. pleuropneumoniae (type strain) & M75074 & - & - & $99 \cdot 7$ & $96 \cdot 2$ \\
\hline CCUG 22229 (genomospecies 1) & AF247722 & - & 70 & $96 \cdot 3$ & $89 \cdot 9$ \\
\hline CCUG 19799 ('haemolytic equuli') & AF247716 & - & 48 & $97 \cdot 5$ & $86 \cdot 9$ \\
\hline A. suis (type strain) & M75071 & 36 & 46 & $98 \cdot 0$ & $87 \cdot 3$ \\
\hline A. ureae (type strain) & M75075 & 37 & - & $98 \cdot 1$ & $87 \cdot 1$ \\
\hline A. arthritidis (type strain) & AF247712 & 43 & - & $97 \cdot 0$ & $86 \cdot 6$ \\
\hline A. hominis (type strain) & L06076 & - & 38 & $97 \cdot 3$ & $88 \cdot 8$ \\
\hline CCUG 16493 (Bisgaard taxon 5) & L06080 & - & - & $95 \cdot 1$ & $81 \cdot 2$ \\
\hline A. muris (type strain) & AF024526 & - & - & $93 \cdot 7$ & $78 \cdot 9$ \\
\hline
\end{tabular}

${ }^{\star}$ Full-length (>1400 nt) 16S rRNA gene sequences were compared with that of the type strain of A. lignieresii (accession no. M75068).

A. lignieresii as $A$. muris or $P$. pneumotropica. By infB comparison, the similarity values of members on the A. equuli branch (omitting A. capsulatus) with the type species of the genus Actinobacillus are in the range 86.9$88 \cdot 8 \%$, the similarity level of A. capsulatus and Bisgaard taxon 8 (which is of unresolved taxonomic position) is $84 \cdot 3-84 \cdot 5 \%$, whereas strains clearly located outside the genus Actinobacillus showed similarity to A. lignieresii of $81 \cdot 7 \%$ or less (Table 2 ).

\section{DISCUSSION}

At present, bacterial systematics relies heavily on comparisons of $16 \mathrm{~S}$ rRNA gene sequences. The method is relatively simple and rapid and can be performed with any bacterial isolate. A number of phylogenetic computer programs is available to do routine allocation to a sequence group of a novel isolate. However, potential problems of both a technical and fundamental nature do exist.

Comparison of 16S rRNA gene sequences must often be carried out with sequences of unequal lengths. While substitutions can be handled easily, either as evenly weighted characters or by assignment of a value according to the type of substitution, it is not easy to score differences in length of sequences. Between the type strains of A. muris and A. lignieresii, a difference in length of nearly $2 \%$ was found in the studied fragment of $16 \mathrm{~S}$ rRNA. This difference is likely to be of phylogenetic significance, but, due to the inability of currently used algorithms to take this into account, the information is ignored. The allowance for gaps in the comparison of sequences most likely underestimates the phylogenetic distance between bacterial strains.

Another problem arises when differences are encountered between the multiple $r r n$ operons in the same bacterial strain. PCR amplification results in an average product, which may be biased towards any of the individual sequences. The method used in the present study does not permit the certain determination of different sequences in separate operons; however, a clear indication of this phenomenon was observed with A. hominis and A. equuli. With the type strain of the latter species, 12 polymorphic positions were found within a stretch of $21 \mathrm{nt}$, which were also seen in the sequence deposited by Berthoud et al. (2002) (GenBank/ EMBL accession no. AF381186). The equivocally determined nucleotides were not seen with all representatives of the various species. It is possible that repeated sequencing would have revealed ambiguities in a larger number of strains, but this was not attempted, as the sequences were accepted on defined criteria and because another sequencing method would be needed in order to identify the individual operons with certainty. The accepted strategy when comparing $16 \mathrm{~S}$ rRNA sequences is to ignore positions with ambiguously determined nucleotides. However, even when accurate information is available, e.g. from wholegenome sequencing projects, it is difficult to make use of the information, as different phylogenies could be inferred 
from different operons. The taxonomic problems associated with $r r n$ operon heterogeneity in the genus Veillonella have recently been addressed (Marchandin et al., 2003).

In general, greater intraspecies variation was observed with inf $B$ sequences than with $16 S$ rRNA gene sequences, with A. capsulatus as the notable exception. Five representatives of this species all carried an identical $\inf B$ sequence, whereas 21 polymorphic positions were encountered in the studied fragment of the 16S rRNA gene. Low similarity (97.9\%) has previously been noted between the near-full-length sequences deposited in databases for the type strain of A. capsulatus and A. capsulatus strain P796 (Olsen et al., 2004). Identical gene sequences in different bacterial strains could indicate intraspecies horizontal spread of a preferred sequence; however, in four positions in the 16S rRNA gene where the type strain differed from three other representatives of A. capsulatus, nucleotides in the fifth strain (P796) were ambiguously determined. It is therefore possible that the apparent differences between strains of $A$. capsulatus reflect, to some extent, differences in the multiple rRNA operons, and further studies on the homogeneity of this species are needed.

DNA hybridization determinations are reported in the literature with decreasing frequency, which underscores the necessity for other molecular methods to be used for validating conclusions based on $16 \mathrm{~S}$ rRNA sequence comparisons. Phylogenetic analysis of DNA sequences from translated genes is an approach with theoretical advantages (Palys et al., 1997). Ideally, the method should be based on a number of universally distributed genes and multiple strains of each taxon should be included to avoid unrepresentative sequences, which may be the result of recombination events. One candidate gene is $\operatorname{infB}$, encoding the translation initiation factor 2 , which has worked well with the genus Haemophilus (Hedegaard et al., 2001).

Using infB sequence comparison, the genus Actinobacillus sensu stricto should include $A$. lignieresii, A. pleuropneumoniae, A. equuli, A. suis, A. ureae, A. arthritidis and A. hominis, plus the unnamed taxa of 'equine lignieresii' (genomospecies 1) and the sorbitol-negative subgroup of Bisgaard taxon 9 (genomospecies 2). These taxa were all related to the type species of the genus at a level above $85 \%$ and constituted a monophyletic cluster by the neighbourjoining, maximum-likelihood and maximum-parsimony methods. With five representatives of $A$. capsulatus and a single strain of Bisgaard taxon 8, the infB sequences obtained were related to the type species of the genus at a level below $85 \%$, and these taxa were positioned with the $P$. pneumotropica cluster by the majority of the phylogenetic methods employed. These taxa may not belong to the genus Actinobacillus sensu stricto.

A. pleuropneumoniae is the cause of porcine necrotizing pleuropneumonia and was originally allocated to the genus Haemophilus as a consequence of its $\mathrm{V}$ factor (NAD) requirement. The description of $\mathrm{V}$ factor-independent strains led to an investigation of its generic position. It was found to be so closely related to A. lignieresii that individual speciation was not justified on the basis of DNA relatedness (Pohl et al., 1983). Because of the differences in host range and pathological lesions, A. lignieresii and A. pleuropneumoniae were upheld as separate species. The close relationship of these species is reflected in their infB sequences (Fig. 1). A single strain of A. pleuropneumoniae (HK356) may have acquired a fragment of the infB gene by recombination with a strain of $A$. lignieresii.

A. lignieresii is associated with characteristic granulomatous lesions in cattle and sheep (Christiansen, 1917; Olsen \& Møller, 2004) and bacteria of indistinguishable phenotype have been isolated from horses and human horse bites (Dibb et al., 1981). It was recently shown that equine isolates of A. lignieresii represent a unique genomospecies (genomospecies 1; Christensen et al., 2002a). This finding was substantiated by infB sequence comparison (cluster 2 in Fig. 1). Two representatives of the sorbitol-negative subgroup of Bisgaard taxon 9 (genomospecies 2) were also located on the A. lignieresii branch (cluster 3). Clusters 1-3 were placed on a common branch by all three phylogenetic methods, yet it is apparent that relatively large differences separate clusters 2 and 3 from cluster 1 . It is therefore possible that the topology of the A. lignieresii branch is not robust and that inclusion of other species or taxa in the comparison will lead to a shift in the position of clusters 2 and 3. Their affiliation to the genus Actinobacillus is, however, supported by both DNA relatedness and nucleotide sequence similarities (Table 2).

A relatively large number of strains of $A$. equuli were included in the study. Haemolytic variants of $A$. equuli have been designated 'equine isolates of $A$. suis' or Bisgaard taxon 11; recently, a reclassification as A. equuli subsp. haemolyticus was proposed (Christensen et al., 2002b). The subspeciation has not been substantiated by $16 \mathrm{~S}$ rRNA (Berthoud et al., 2002) or infB sequences (Fig. 1). The differences in $\inf B$ sequence between the 12 haemolytic and non-haemolytic strains of $A$. equuli (cluster 4) were comparable with the differences encountered between the 14 representatives of cluster 1 . However, in contrast to the latter cluster, A. equuli was not well separated from the other species of its branch, particularly A. suis (Fig. 1). A similar result was seen in a study of near-full-length $16 \mathrm{~S}$ rRNA sequences of eight strains of $A$. equuli together with representative taxa of the remaining part of the genus Actinobacillus. The phylogeny was poorly resolved and representatives of $A$. equuli were scattered in the dendrogram among other Actinobacillus species (Christensen et al., 2002b).

Four other species on the A. equuli branch, A. suis, A. ureae, $A$. arthritidis and $A$. hominis, were very homogeneous with respect to their $\inf B$ sequences. These species were each represented by two to seven isolates. Because of the restricted sample size, it is not clear whether the apparent similarity of these species is real. 
A. capsulatus presents a particular taxonomic problem. A thorough investigation of DNA relatedness on multiple strains has shown this species to be a true member of the genus Actinobacillus (Escande et al., 1984). In contrast, analysis based on 16 rRNA sequences of five strains showed that the species constituted a separate cluster only remotely related to Actinobacillus sensu stricto (Olsen et al., 2004). Some additional strains were included in this study and the peculiar sequence of the $16 \mathrm{~S}$ rRNA gene was confirmed. In the studied fragment, the type strains of A. capsulatus, A. muris and $P$. pneumotropica were equally different from that of A. lignieresii (Table 2); however, the type strains of A. muris and $P$. pneumotropica carried a $16 \mathrm{~S}$ rRNA sequence that was considerably shorter (429 nt) than the $435 \mathrm{nt}$ observed with all species on the $A$. equuli branch, including $A$. capsulatus. With respect to infB sequence, $A$. capsulatus took up an intermediate position (Table 2) and, although the cluster was placed on the A. equuli branch by the neighbour-joining method (Fig. 1), this was not the case with the maximum-likelihood and maximum-parsimony methods (dendrograms not shown). Further investigations are needed in order to clarify the taxonomic position of A. capsulatus.

Analysis based on comparison of $16 \mathrm{~S}$ rRNA sequences has clustered the duck isolate CCUG 28015 and the reference strain of Bisgaard taxon 8 with Actinobacillus sensu stricto (Olsen et al., 2004). This inclusion into the genus was not apparent from investigations of infB sequences (Fig. 1; Table 2). The taxonomic position of these candidate species awaits analysis of a greater number of representative strains. Bisgaard taxon 5, included in the genus by anecdotal evidence, was clearly located outside the genus Actinobacillus sensu stricto by infB sequence comparison (Fig. 1; Table 2).

A large number of provisional taxa and species of the family Pasteurellaceae have been described in recent years, and the family is likely to undergo rearrangement and reclassification in the future. If relatively high levels of similarity are insisted upon within individual genera, a large number of genera will be needed in order to encompass the diversity of the family. Using infB sequence comparison, an $85 \%$ similarity demarcation line appeared appropriate for delineation of the genus Actinobacillus sensu stricto. Further data on DNA sequence similarity in the genus and in the family are needed before taxonomic conclusions are drawn.

\section{REFERENCES}

Berthoud, H., Frey, J. \& Kuhnert, P. (2002). Characterization of Aqx and its operon: the hemolytic RTX determinant of Actinobacillus equuli. Vet Microbiol 87, 159-174.

Brosius, J., Palmer, M. L., Kennedy, P. J. \& Noller, H. F. (1978). Complete nucleotide sequence of a $16 \mathrm{~S}$ ribosomal RNA gene from Escherichia coli. Proc Natl Acad Sci U S A 75, 4801-4805.

Christensen, H., Bisgaard, M., Angen, Ø. \& Olsen, J. E. (2002a). Final classification of Bisgaard taxon 9 as Actinobacillus arthritidis sp. nov. and recognition of a novel genomospecies for equine strains of Actinobacillus lignieresii. Int J Syst Evol Microbiol 52, 1239-1246.

Christensen, H., Bisgaard, M. \& Olsen, J. E. (2002b). Reclassification of equine isolates previously reported as Actinobacillus equuli, variants of A. equuli, Actinobacillus suis or Bisgaard taxon 11 and proposal of $A$. equuli subsp. equuli subsp. nov. and $A$. equuli subsp. haemolyticus subsp. nov. Int J Syst Evol Microbiol 52, 1569-1576.

Christiansen, M. (1917). En ejendommelig pyaemisk lidelse hos faar. Maanedsskr Dyrlaeg 29, 449-458 (in Danish).

Dewhirst, F. E., Paster, B. J., Olsen, I. \& Fraser, G. J. (1992). Phylogeny of 54 representative strains of species in the family Pasteurellaceae as determined by comparison of $16 \mathrm{~S}$ rRNA sequences. J Bacteriol 174, 2002-2013.

Dibb, W. L., Digranes, A. \& Tønjum, S. (1981). Actinobacillus lignieresii infection after a horse bite. $\mathrm{Br}$ Med J 283, 583-584.

Escande, F., Grimont, F., Grimont, P. A. D. \& Bercovier, H. (1984). Deoxyribonucleic acid relatedness among strains of Actinobacillus spp. and Pasteurella ureae. Int J Syst Bacteriol 34, 309-315.

Felsenstein, J. (1995). PHYLIP (phylogenetic inference package), version 3.57c. Department of Genetics, University of Washington, Seattle, WA, USA.

Fleischmann, R. D., Adams, M. D., White, O. \& 37 other authors (1995). Whole-genome random sequencing and assembly of Haemophilus influenzae Rd. Science 269, 496-512.

Friis-Møller, A., Christensen, J. J., Fussing, V., Hesselbjerg, A., Christiansen, J. \& Bruun, B. (2001). Clinical significance and taxonomy of Actinobacillus hominis. J Clin Microbiol 39, 930-935.

Fussing, V., Paster, B. J., Dewhirst, F. E. \& Poulsen, L. K. (1998). Differentiation of Actinobacillus pleuropneumoniae strains by sequence analysis of $16 \mathrm{~S}$ rDNA and ribosomal intergenic regions, and development of a species specific oligonucleotide for in situ detection. Syst Appl Microbiol 21, 408-418.

Hedegaard, J., Steffensen, S. A., Nørskov-Lauritsen, N., Mortensen, K. K. \& Sperling-Petersen, H. U. (1999). Identification of Enterobacteriaceae by partial sequencing of the gene encoding translation initiation factor 2. Int J Syst Bacteriol 49, 1531-1538.

Hedegaard, J., Okkels, H., Bruun, B., Kilian, M., Mortensen, K. K. \& Norskov-Lauritsen, N. (2001). Phylogeny of the genus Haemophilus as determined by comparison of partial infB sequences. Microbiology 147, 2599-2609.

Kilian, M. (1976). A taxonomic study of the genus Haemophilus, with the proposal of a new species. J Gen Microbiol 93, 9-62.

Kilian, M., Nicolet, J. \& Biberstein, E. L. (1978). Biochemical and serological characterization of Haemophilus pleuropneumoniae (Matthews and Pattison 1961) Shope 1964 and proposal of a neotype strain. Int J Syst Bacteriol 28, 20-26.

Kimura, M. (1980). A simple method for estimating evolutionary rates of base substitutions through comparative studies of nucleotide sequences. J Mol Evol 16, 111-120.

Krieg, N. R. \& Garrity, G. M. (2001). On using the manual. In Bergey's Manual of Systematic Bacteriology, 2nd edn, vol. 1, pp. 15-19. Edited by D. R. Boone \& R. W. Castenholz. New York: Springer.

Marchandin, H., Teyssier, C., Siméon de Buochberg, M., JeanPierre, H., Carriere, H. \& Jumas-Bilak, E. (2003). Intra-chromosomal heterogeneity between the four $16 \mathrm{~S}$ rRNA copies in the genus Veillonella: implications for phylogeny and taxonomy. Microbiology 149, 1493-1501.

May, B. J., Zhang, Q., Li, L. L., Paustian, M. L., Whittam, T. S. \& Kapur, V. (2001). Complete genomic sequence of Pasteurella multocida, Pm70. Proc Natl Acad Sci U S A 98, 3460-3465. 
Mutters, R., Mannheim, W. \& Bisgaard, M. (1989). Taxonomy of the group. In Pasteurella and Pasteurellosis, pp 3-34. Edited by C. Adlam \& J. M. Rutter. London: Academic Press.

Olsen, I. \& Møller, K. (2004). Genus Actinobacillus Brumpt 1910, $849^{\mathrm{AL}}$. In Bergey's Manual of Systematic Bacteriology, 2nd edn, vol. 2. Edited by D. J. Brenner, N. R. Krieg, J. T. Staley \& G. M. Garrity. New York: Springer (in press).

Olsen, I., Dewhirst, F. E., Paster, B. J. \& Busse, H.-J. (2004). Family Pasteurellaceae Pohl 1981, 382 VP. In Bergey's Manual of Systematic Bacteriology, 2nd edn, vol. 2. Edited by D. J. Brenner, N. R. Krieg, J. T. Staley \& G. M. Garrity. New York: Springer (in press).

Palys, T., Nakamura, L. K. \& Cohan, F. M. (1997). Discovery and classification of ecological diversity in the bacterial world: the role of DNA sequence data. Int J Syst Bacteriol 47, 1145-1156.
Phillips, J. E. (1984). Genus III. Actinobacillus Brumpt 1910, $849^{\mathrm{AL}}$. In Bergey's Manual of Systematic Bacteriology, pp. 570-575. Edited by N. R. Krieg \& J. G. Holt. Baltimore: Williams \& Wilkins.

Pohl, S., Bertschinger, H. U., Frederiksen, W. \& Mannheim, W. (1983). Transfer of Haemophilus pleuropneumoniae and the Pasteurella haemolytica-like organism causing porcine necrotic pleuropneumonia to the genus Actinobacillus (Actinobacillus pleuropneumoniae comb. nov.) on the basis of phenotypic and deoxyribonucleic acid relatedness. Int J Syst Bacteriol 33, 510-514.

Thompson, J. D., Gibson, T. J., Plewniak, F., Jeanmougin, F. \& Higgins, D. G. (1997). The CLUSTAL_X windows interface: flexible strategies for multiple sequence alignment aided by quality analysis tools. Nucleic Acids Res 25, 4876-4882. 